\title{
Adrenal ganglioneuroblastoma presenting as an acute cerebellar syndrome
}

\author{
Jithangi Wanigasinghe ${ }^{1}$, Mahendra Somathilake ${ }^{2}$, Dharmasri Jayawardena ${ }^{3}$
}

Sri Lanka Journal of Child Health, 2013; 42(2): 99-100

(Key words: Adrenal ganglioneuroblastoma; acute cerebellar syndrome; child)

Commonest cause of acute cerebellar dysfunction in children is post viral cerebellitis. Unlike in adults, where isolated acute cerebellar dysfunction is a common paraneoplastic manifestation, it is only rarely reported in children ${ }^{1}$. We report a child presenting with this syndrome secondary to an underlying ganglioneuroblastama.

\section{Case report}

A 2 year old boy presented with acute onset unsteady gait, intention tremor and dysarthria. Examination revealed marked truncal and limb ataxia, bilateral hypotonia, past pointing, a disabling intention tremor and staccato type dysarthria. Dysdiadochokinesia could not be elicited reliably. He did not have any abnormal eye movements i.e. nystagmus or opsoclonus. He was reported to be difficult to console and irritable and had drooling of saliva. Functionally he was markedly disabled due to the ataxia and tremor. There was no history of a preceding viral infection, immunisation or recent drug ingestion. There were no features of an ongoing central nervous system (CNS) infection.

Cerebrospinal fluid assessment was negative for infection including tuberculosis. No cytoprotein dissociation was noted. His MRI scan of brain was normal. As he was markedly symptomatic he was treated with a short course of high dose methyl prednisolone to ameliorate symptoms due to possible post viral cerebellitis. This resulted in a significant improvement of his cerebellar symptoms and signs for next four weeks. However he re-presented one month later due to gradual reappearance of acute cerebellar symptoms. He could not even sit unsupported and he had several encephalopathic features such as irritability, frequent biting and aggressiveness. There was still no opsoclonus or myoclonus or nystagmus. No seizure activity or any other episodic fluctuations of heart rate/blood pressure or other neurological

\footnotetext{
${ }^{1}$ Senior Lecturer in Paediatrics, Department of Paediatrics, Faculty of Medicine, University of Colombo, ${ }^{2}$ Senior Registrar in Paediatric Oncology, ${ }^{3}$ Consultant Paediatric Surgeon, Lady Ridgeway Hospital, Colombo
}

(Received on 31 July 2012: Accepted on 28 September 2012) deficits were noted. Repeat MRI brain scan showed no abnormalities. Sleep EEG did not show evidence of electrical status epilepticus in sleep. The urine screen for aminoacids was negative. Serum lactic acid, urinary VMA levels and ammonia were normal.

In spite of absence of opsoclonus, USS of abdomen was performed to exclude rare possibility of underlying neuroblastoma. This detected a $0.7 \times 1$ $\mathrm{x} 1 \mathrm{~cm}$ cystic mass in the suprarenal gland, confirmed later in $\mathrm{CT}$ scan of abdomen. Bone marrow aspiration was negative. He underwent laparoscopic adrenalectomy which was followed by immediate reduction of his encephalopathic behaviour. The drooling and ataxia improved gradually over the next two months. The surgical histopathology revealed a ganglioneuroblastoma. As the margins were incompletely resected he was treated with six cycles of chemotherapy with cyclophosphamide and vincristine. Follow up till one year post chemotherapy confirmed absence of any recurrence.

\section{Discussion}

Paraneoplastic clinical manifestations are clinical symptoms and signs that occur, remote to the tumour site or metastasis. It is reported in association with any type of non CNS malignant tumours . The pathogenesis of these manifestations is attributed to the effects of auto-antibodies that are produced by the body in response to the tumour. These antibodies adversely affect function of distant organs thus resulting in varying symptomatology. In children, the most common paraneoplastic manifestation is the syndrome of paraneoplastic opsoclonus myoclonus $(\mathrm{POM})^{2}$. This is reported almost exclusively in young children (6 months - 3 years) and affects $2 \%-3 \%$ of those with neuroblastoma. The classical clinical features include a bizarre ocular movement called "opsoclonus" and an erratic multifocal myoclonic component that gives rise to the ataxia. Other rare clinical manifestations seen concurrently with POM include speech disturbances, focal neurological signs, urinary signs and drooling ${ }^{2}$. Our patient was detected to have a ganglioneuroblastoma, one of the three tumours originating from neuroblastic cells showing partially matured, differentiating cells. 
In our patient there was no opsoclonus, only features of pure cerebellar dysfunction. This was the reason for consideration of possible post viral cerebellitis and the short delay in establishing the diagnosis. Awareness of this rare association of isolated cerebellar syndrome as a paraneoplastic manifestation in children $^{3,4}$ will facilitate appropriate and timely interventions.

\section{References}

1. Mitchell WG, et al. Opsoclonus-ataxia caused by childhood neuroblastoma: developmental and neurologic sequelae. Pediatrics 2002; 109(1): 86-98.

2. Pranzatelli MR. The neurobiology of the opsoclonus-myoclonus syndrome. Clinical Neuropharmacology 1992. 15(3): 186-228. http://dx.doi.org/10.1097/00002826199206000 $\underline{-00002}$

3. Solomon GE, Chutorian AM. Opsoclonus and occult neuroblastoma. New England Journal of Medicine 1968. 279(9): p. 475-7.

http://dx.doi.org/10.1056/NEJM196808292790 $\underline{907}$

4. Balakrishnan V, Simpson DA. Cerebellar syndromes in occult neuroblastomas. Australian Paediatric Journal 1980. 16(3): 191-3. 